\title{
SPATIOTEMPORAL REORGANIZATION OF GROWTH RATES IN THE EVOLUTION OF ONTOGENY
}

\author{
Miriam L. Zelditch, ${ }^{1}$ H. David Sheets, ${ }^{2}$ And William L. FinK ${ }^{3}$ \\ ${ }^{1}$ Museum of Paleontology, University of Michigan, Ann Arbor, Michigan 48109 \\ E-mail: zelditch@umich.edu \\ ${ }^{2}$ Department of Physics, Canisius College, Buffalo, New York 14208 \\ E-mail: sheets@canisus.edu \\ ${ }^{3}$ Museum of Zoology and Department of Biology, University of Michigan, Ann Arbor, Michigan 48109 \\ E-mail:wfink@umich.edu
}

\begin{abstract}
Heterochrony, evolutionary changes in rate or timing of development producing parallelism between ontogeny and phylogeny, is viewed as the most common type of evolutionary change in development. Alternative hypotheses such as heterotopy, evolutionary change in the spatial patterning of development, are rarely entertained. We examine the evidence for heterochrony and heterotopy in the evolution of body shape in two clades of piranhas. One of these is the sole case of heterochrony previously reported in the group; the others were previously interpreted as cases of heterotopy. To compare ontogenies of shape, we computed ontogenetic trajectories of shape by multivariate regression of geometric shape variables (i.e., partial warp scores and shape coordinates) on centroid size. Rates of development relative to developmental age and angles between the trajectories were compared statistically. We found a significant difference in developmental rate between species of Serrasalmus, suggesting that heterochrony is a partial explanation for the evolution of body shape, but we also found a significant difference between their ontogenetic transformations; the direction of the difference between them suggests that heterotopy also plays a role in this group. In Pygocentrus we found no difference in developmental rate among species, but we did find a difference in the ontogenies, suggesting that heterotopy, but not heterochrony, is the developmental basis for shape diversification in this group. The prevalence of heterotopy as a source of evolutionary novelty remains largely unexplored and will not become clear until the search for developmental explanations looks beyond heterochrony.
\end{abstract}

Key words.-Allometry, geometric morphometrics, growth, heterochrony, heterotopy, ontogeny, teleosts.

Received August 17, 1999. Accepted January 23, 2000.

Heterochrony refers to evolutionary change in developmental rate or timing that results in parallelism between ontogeny and phylogeny (Gould 1977; p. 2). This parallelism is evident in a close similarity between the morphologies of putative ancestors and descendants at different ages; for example, the descendant adult might strongly resemble the ancestral juvenile. Hundreds of studies report empirical evidence of heterochrony in a diverse array of taxa, including plants (e.g., Lord and Hill 1987; Bateman 1991; Rutishauser 1997), trilobites (e.g., McNamara 1978; Edgecombe and Chatterton 1987; Ramskold 1988), and vertebrates (e.g., Alberch and Alberch 1981; Bemis 1984; Winterbottom 1990). Based on this literature, it would seem that heterochrony is an especially prevalent type of evolutionary change in development. It is certainly detected more often than others, such as heterotopy, which refers to evolutionary change in the spatial patterning of development (Wray and McClay 1989; Zelditch and Fink 1996).

The apparent predominance of heterochrony in the literature may reflect its predominance in nature. Heterochrony may indeed be an especially common phenomenon, as argued by some workers (Alberch and Alberch 1981; Raff and Kaufman 1983; McKinney 1988; McKinney and McNamara 1991; Reilly et al. 1997). Yet, others find no a priori reason for the predominance of heterochrony, suggesting that heterochrony and heterotopy may be equally common (Wray and McClay 1989; Zelditch and Fink 1996). According to this alternative view, heterochrony may seem so common only because it has been the focus of concerted attention for two decades, whereas the hypothesis of heterotopy is rarely even considered, much less tested. Also, some workers have expanded the definition of heterochrony to the point that virtually any evolutionary change in the ontogeny of form would qualify as heterochrony (e.g., McKinney and McNamara 1991). Moreover, some studies might emphasize the evidence for heterochrony even when more complex explanations are suggested by the data. In such cases, comparisons might reveal a similarity between ancestral and descendant morphologies (at different ages), but not to the extent required by Gould's metric for heterochrony: the ratio of sizes or ages at which the two morphologies are geometrically similar (Gould 1977, p. 241). Even though a hypothesis of heterochrony would not be rejected on those grounds, that hypothesis does not fully explain the data because it fails to account for the deviation from geometric similarity.

Heterotopy could explain why morphologies differ by more than expected under a hypothesis of heterochrony. Under a hypothesis of heterotopy, taxa do not simply progress more or less rapidly along the same ontogenetic trajectory, they diverge in the spatial pattern of growth. If heterotopy and heterochrony both occur, taxa will progress more or less rapidly along divergent ontogenetic trajectories. Such a combination of processes, or even heterotopy alone, may be difficult to detect empirically when morphology is described in simple terms, for example, by degree of ossification or by the length of a structure. That is because such one-dimensional features would always appear to be more or less developed in one taxon; there is no other way in which they could possible differ. Being one-dimensional, these features necessarily both develop and evolve along the same dimension so they always suggest parallelism between ontogeny and phylogeny. Moreover, comparisons between them with 
respect to age can reveal changes of only three sorts: (1) age at onset of development; (2) rate of development; or (3) age at offset of development (McKinney and McNamara 1991). No other possibilities are allowed by the geometry of the data. But complex morphologies are not one-dimensional and can reveal more kinds of developmental patterns, including changes in the spatial pattern of development. Such changes in spatial pattern can be discerned by comparing spatially distributed growth rates, something rarely done in comparative studies of allometry that concentrate on the magnitudes of rates or intercepts. But ontogenetic allometries have locations on the organism, not just rates, and there is no reason to believe that these spatial patterns are historically conserved while rates evolve. Spatial patterning may be just as labile as timings and rates.

Should morphology typically evolve by changing both spatial and temporal aspects of ontogeny, as proposed by Zelditch and Fink (1996), heterochrony is rarely an adequate explanation (Zelditch and Fink 1996). This conjecture was based primarily on first principles, specifically, from the premise that development occurs in both space and time. However, it is not known whether both aspects of ontogeny are equally labile in evolution and arguments from first principles are unconvincing without well-supported models for the relevant processes, which do not exist for late stages of skeletal development. For that reason, we need empirical tests of the expectation that morphology typically evolves by the combination of these two processes.

Herein we test the hypothesis that evolutionary change occurs by a combination of heterochrony and heterotopy. To do so, we focus on two clades of piranhas. One includes the sole case of heterochrony reported in piranhas, Serrasalmus elongatus (Fink 1989); the other is the sole case in which heterochrony was explicitly rejected as an explanation for the evolution of form, Pygocentrus (Fink and Zelditch 1995). Both cases seem to contradict our hypothesis because each one suggests that only a single aspect of growth evolves, rate in the case of $S$. elongatus, spatial patterning in the case of Pygocentrus. But both groups need reexamination. The previous analysis of $S$. elongatus oversimplified form, basing the analysis on one dimension, overall body depth. This feature was singled out because adults of $S$. elongatus are distinctively shallow bodied, resembling juveniles of its clade. Not surprisingly, a comparison of rates of deepening showed that $S$. elongatus develops slowly in this feature compared to another member of the clade, $S$. gouldingi. But body depth is only one aspect of form; we need to determine whether the ontogeny, as a whole, is reduced in rate, but otherwise conserved. The previous analysis is also suspect because developmental rates were not compared, nor were the ontogenies statistically compared to each other (Fink 1989). The inference about Pygocentrus was based on a phylogenetic analysis of ontogenies of shape variables, an approach that has been criticized as univariate (Rohlf 1998a), but which may be more properly criticized as informally multivariate, relying as it does on phylogenetic correlations among variables. In this reexamination we compare rates of development and use more formal methods for comparing the directions of ontogenetic change.

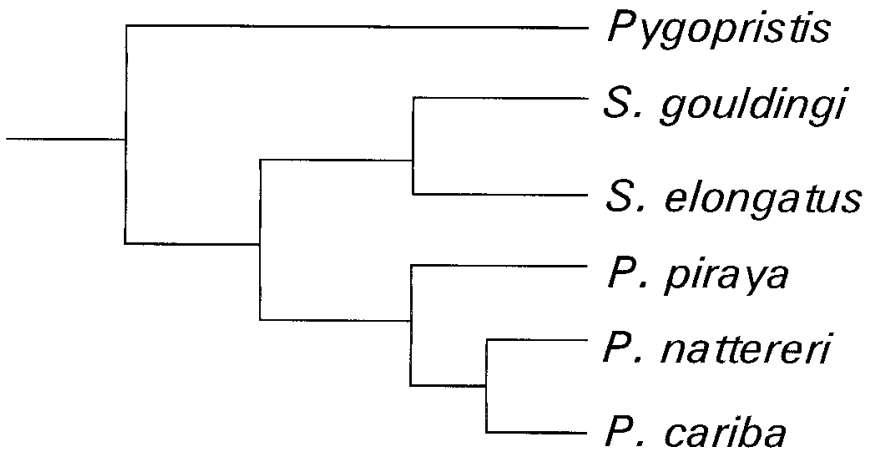

FIG. 1. Cladogram of piranhas used in this study.

\section{Materials And Methods}

Data

Our samples comprise ontogenetic series drawn from natural populations of six species: Pygopristis denticulata $(n=$ 32, Standard Length [SL] = 23.1-182.1 mm); Serrasalmus gouldingi $(n=38, \mathrm{SL}=28.7-282.84 \mathrm{~mm}) ;$ S. elongatus $(n$ $=46,=$ SL 28.4-227.0 mm); Pygocentrus piraya $(n=34$, $=\mathrm{SL} 16.0-322.0 \mathrm{~mm}) ;$ P. cariba $(n=31, \mathrm{SL}=24.0-193.0$ $\mathrm{mm})$; and $P$. nattereri $(n=89, \mathrm{SL}=20.5-277.5 \mathrm{~mm})$. Phylogenetic relationships among them are shown in Figure 1. In our prior comparative analyses, we use Pygopristis as the outgroup for comparing ontogenies (Fink and Zelditch 1995, 1996). However, the two species of Serrasalmus have a distinctive juvenile morphology, so Pygopristis does not represent the primitive juvenile morphology for that clade. Nor does the juvenile morphology of Pygopristis closely resemble the juvenile morphology of species of Pygocentrus. Thus, we cannot estimate the ancestral juvenile morphology for either Serrasalmus or Pygocentrus by outgroup comparison. This is important for testing the hypothesis of heterochrony because the hypothesis presupposes that the ancestral juvenile morphology is conserved. Thus, in testing hypotheses of heterochrony, we compare the two species of Serrasalmus to each other, and the three species of Pygocentrus to each other. However, because it is possible that the direction of juvenile ontogeny is conserved even though juvenile morphology is modified, we include Pygopristis in the comparative analyses of ontogeny.

Lacking information on chronological age, we use body size as our estimate of developmental age. The use of size rather than age is controversial (see Blackstone 1987; Strauss 1987; McKinney 1988; Schweitzer and Lohmann 1990). However, the arguments about the merits of these two indicators of developmental age typically focus on the issue of using them to diagnose modes of heterochrony (e.g., neoteny, progenesis). Attempts to identify types of heterochronic perturbations from size data alone are problematic because the relationship between size and age may evolve. However, we are not presently concerned with such identifications. Rather, we are concerned with the evidence for heterochrony, per se, and that evidence requires similarity of ontogenetic transformations. It is therefore crucial to reconstruct those transformations accurately. An empirical study determined that estimates of developmental progress based on chronological 
age are both more sensitive to temperature and more intrinsically variable than those based on size (measured as the logarithm of length) in fishes (Fuiman et al. 1998), so estimates of developmental age based on size may be more reliable than those based on chronological age.

Size might be a poor proxy for developmental age if size and developmental age are dissociated in their evolution. In the case of our taxa, this does not appear to be the case; all taxa begin posttransformational growth at a similar size, reach adult body shape at a similar size, and, as determined in this study, all show a linear relationship between shape and size throughout this interval. Certainly, it is possible that compensatory modifications in rate and duration occur such that rates of growth are decreased relative to time, whereas the duration of that interval is extended in time, and the rate of development (relative to growth) is unmodified. The result would be an unmodified size at maturity and an unmodified rate of development relative to size, but the duration of posttransformational growth would be different. We are unable to detect compensatory changes without information on chronological age; nevertheless, we can identify paedomorphic morphologies (i.e., the derived adult morphology resembles the primitive juvenile morphology) and peramorphic morphologies (i.e., the derived adult morphology is predicted by extrapolating the primitive developmental trajectory) and compare rates of development with respect to developmental age.

Our analyses of ontogenetic change in morphology are based on landmarks, discrete points that are recognizable and arguably homologous on all specimens in the study (Fig. 2). These landmarks were chosen to provide the most comprehensive and even coverage possible using external landmarks. Because these individuals are laterally flattened, little information is lost or distorted when the specimens are projected onto a plane. All landmarks were digitized by one person (W. L. Fink) on the left side of each specimen.

\section{Morphometric Methods}

To examine the ontogeny of shape, we use geometric morphometrics. Geometric methods have two distinct advantages over more conventional methods for the analysis of heterochrony and heterotopy. First, the original models for heterochrony were framed in explicitly geometric terms (Gould 1977; Alberch et al. 1979). Inferences based on these models, if applied out of the context of the original dimensions, can be misleading (Godfrey and Sutherland 1996). Second, geometric methods can analyze spatial relationships among landmarks, which is obviously useful for studies of ontogenetic spatial patterning. In our analyses, we use two types and shape variables, shape coordinates and partial warps. Landmark locations are transformed to shape coordinates by selecting two points (landmarks 1 and 7) to serve as the endpoints of a baseline as assigning them the coordinates $(0,0)$ and $(1,0)$. All specimens are then transformed to the same baseline orientation and length, a transformation that does not alter the shape of landmark configurations (Bookstein 1986, 1991). The coordinates that are not fixed are interpreted as the third vertex of a triangle drawn to the baseline. Partial warps, calculated from the shape coordinates, are geomet-

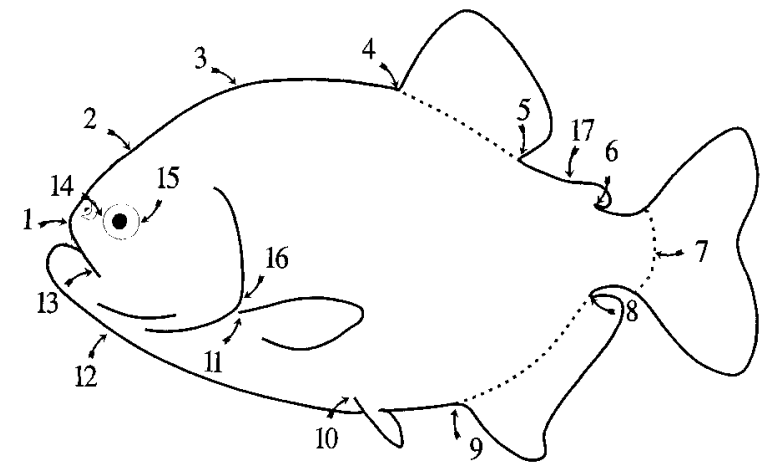

FIG. 2. Landmarks; 1, snout tip, anteroventral junction of anteriomedial borders of premaxillaries; 2 , anterior border of epiphyseal bridge bone at the dorsal midline (an insect pin was inserted into the top of the cranium to detect the border and was left in place for digitization); 3 , posterior tip of supraoccipital bone where it lies adjacent to epaxial musculature and the median dorsal septum; 4, dorsal fin origin, not including anterior modified fin rays, marking the anterior junction of the fin and the dorsal body midline; 5, posterior end of dorsal fin base at the dorsal body midline; 6 , posterior end of adipose fin base, where it joins with the skin of the posterior back on the dorsal midline; 7, posterior border of hypural bones (identified as the bending axis of the caudal fin base); 8 , posterior end of anal fin base, at the ventral midline; 9, anal fin origin, marking the junction of the fin and the ventral body midline; 10 , pelvic fin insertion, where the fin projects laterally from the pelvic girdle; 11, pectoral fin insertion, where the pectoral fin extends laterally from its joint with the pectoral girdle; 12 , mandible/ quadrate joint (usually marked by an insect pin placed in the middle of the joint), marking the junction between the lower jaw and "face"'; 13, posterior border of maxillary bone, where it intersects the third infraorbital (cheek) bone; 14, anterior border of bony orbit, along the horizontal body axis; 15 , posterior bony border of orbit, along the horizontal body axis; 16, posterior border of bony operculum, at most posterior point from the snout tip.

rically orthogonal components of a deformation modeled by the thin-plate spline (Bookstein 1989, 1991). The deformation is decomposed into two components, the uniform (affine) and the nonuniform. The uniform component describes changes in form that are geometrically uniform over the whole body such that each small square of a starting grid superimposed on the undeformed shape is transformed into the same parallelogram in the same orientation. The nonuniform component is further decomposed into a series of progressively more localized dimensions called partial warps (for comparatively nontechnical accounts of these variables, see Swiderski 1993; Zelditch and Fink 1995). As a measure of size, we use centroid size, the square root of the summed squared distances between each landmark and the centroid of the form; it is the sole size variable independent of shape in the absence of allometry (Bookstein 1991).

The ontogenetic change in shape of each species was analyzed by multivariate regression of shape on size. Each full set of shape variables (i.e., the full set of shape coordinates) and the full set of partial warp scores (including scores on the uniform component) was regressed on centroid size. The null hypothesis is that shape develops isometrically. Because most ontogenetic shape change in our samples occurs early in ontogeny, we use log-transformed centroid size as our size variable. Statistical analyses were done using Systat (SPSS 1990); graphical depictions of ontogenetic allometries were 
obtained from tpsRegr (Rohlf 1998b; available at http:// life.bio.sunysb.edu/morph).

Comparisons among ontogenies were done by MANCO$\mathrm{VA}$, testing the null hypothesis of homogeneity of slopes. When slopes differ, three possibilities need to be examined: (1) taxa differ in the rate of development along the same ontogenetic trajectory; (2) taxa differ in their ontogenetic trajectories of shape (but not in their rate of progress along these ontogenetic trajectories); and (3) taxa differ in both rate and direction of ontogenetic transformations. To distinguish among these, we estimated and compared both developmental rates and ontogenetic trajectories of shape.

To estimate the rate of development, we calculated a rate of change in shape for each species, which corresponds to the rate of development as defined by Gould (1977). The estimate is based on a morphometric distance between each specimen and the average juvenile, calculated as a Procrustes distance, the generally accepted metric for shape dissimilarity in geometric morphometrics (Bookstein 1996; Dryden and Mardia 1998). Procrustes distances were regressed on logtransformed centroid size to calculate the rate of divergence away from the juvenile for each taxon. Because the relationship between Procrustes distance and log-transformed centroid size is close to linear, these can be statistically compared by MANCOVA.

To determine if taxa differ in the vectors of ontogenetic shape change, we computed angles between vectors of ontogenetic allometric coefficients normalized to unit length. These angles are arc cosines of vector correlations. The null hypothesis is that the angle is zero, which is equivalent to the hypothesis of a conserved ontogenetic trajectory of shape. To test this hypothesis statistically, we formed confidence intervals for the angle by a resampling procedure (Efron and Tibshirani 1993). The basic approach is to compare the angle between ontogenetic vectors of two species to angles between ontogenetic vectors from one species. The angle between the vectors of two different species would be considered statistically significant if it exceeds that calculated from samples drawn from a single species.

To obtain confidence intervals for the angles, we formed bootstrapped sets by bootstrapping the residuals of the multivariate regression of landmark coordinates or partial warp scores on log-transformed centroid size. Each sample in the original dataset produced a set of residuals, one per shape coordinate or partial warp. Each sample point in a bootstrap set was formed by using the multivariate regression coefficients to calculate the predicted landmark or partial warp values for a given log-transformed centroid size from the dataset and then adding to these predicted values a set of residuals drawn at random with replacement from the set of residuals. Each sample point in the original dataset thus contributed a set of residuals, one per landmark or partial warp, which were bootstrapped as a complete set, preserving any covariance structure (across landmarks or partial warps) present in the residuals. The same calculation was also done by bootstrapping residuals independently, partial warp by partial warp or coordinate by coordinate, rather than as sets, thus destroying any correlation among residuals across landmarks or partial warps. The bootstrapping of sets of residuals produced a consistently larger confidence interval than the in- dependent bootstrapping of individual landmark or partial warp residuals, so the larger ranges produced by bootstrapping sets of residuals were used in this study to yield a conservative estimate of the range of within-species variation in angle. The angle between ontogenetic vectors produced between two species was judged significant only if it exceeded the $95 \%$ confidence interval of within-species range of angles obtained from the distribution of bootstrapped datasets at comparable sample sizes. Resampling tests were performed using functions written in MATLAB (Mathworks 1997).

To identify variables that differentiate ontogenies, we examined differences between the ontogenetic trajectories, expressed both in shape coordinates and partial warp scores, in the amplitude and direction of the individual two-dimensional variables, that is, the two shape coordinates for each landmark and the two directions for each partial warp. Amplitudes were compared to within-species $95 \%$ confidence range of amplitudes for that particular variable, determined by the same bootstrapping approach as discussed earlier. For the difference in the amplitude to be judged significant, each of the two species in the comparison had to have an amplitude of the two-dimensional variable outside the $95 \%$ confidence interval of within-species range of amplitude of the other species in the comparison. Similarly, the difference in the angles of two-dimensional variable between the two species had to be outside the $95 \%$ confidence interval of within-species differences in angle generated by bootstrap sets of both species. The statistical comparisons of individual landmarks, as well as those of individual partial warps, do not imply that they are individually meaningful in any biological sense. The direction of change is inferred from the full set of variables that differentiate ontogenies.

\section{RESUlts}

The null hypothesis of isometric growth is rejected $(P<$ 0.001 ) for the five species with samples large enough for statistical analysis (this excludes $P$. cariba); ontogenetic shape changes are depicted by changes in the location of shape coordinates (Fig. 3) and as deformations (Fig. 4). Comparisons of the slopes between the outgroup ( $P$. denticulata) and each species of Pygocentrus reveal significant heterogeneity $\left(P<1.0 \times 10^{-6}\right.$ in all three comparisons $)$. Moreover, the three species of Pygocentrus differ in slope $(P=4.18 \times$ $\left.10^{-12}\right)$; no two of them share a common ontogenetic transformation $\left(P=1 \times 10^{-6}\right.$ for the comparison between $P$. piraya and $P$. nattereri; $P=3.541 \times 10^{-4}$ for the comparison between $P$. piraya and $P$. cariba; and $P=3.51 \times 10^{-8}$ for the comparison between $P$. cariba and $P$. nattereri). The two Serrasalmus also differ significantly; given the visually obvious difference in the ontogeny of body depth, comparisons were made both including and excluding the uniform component of body deepening; the slopes differ whether that component is included $\left(P=4.08 \times 10^{-6}\right)$ or excluded $(P=$ $\left.1.5 \times 10^{-6}\right)$.

Developmental rate differs significantly between $S$. gouldingi and $S$. elongatus $\left(P=9.0 \times 10^{-16}\right)$, but the rate of development in $S$. elongatus does not seem dramatically less than in other piranhas (Table 1). Developmental rate seems conservative in Pygocentrus $(P=0.496)$. The rate of devel- 

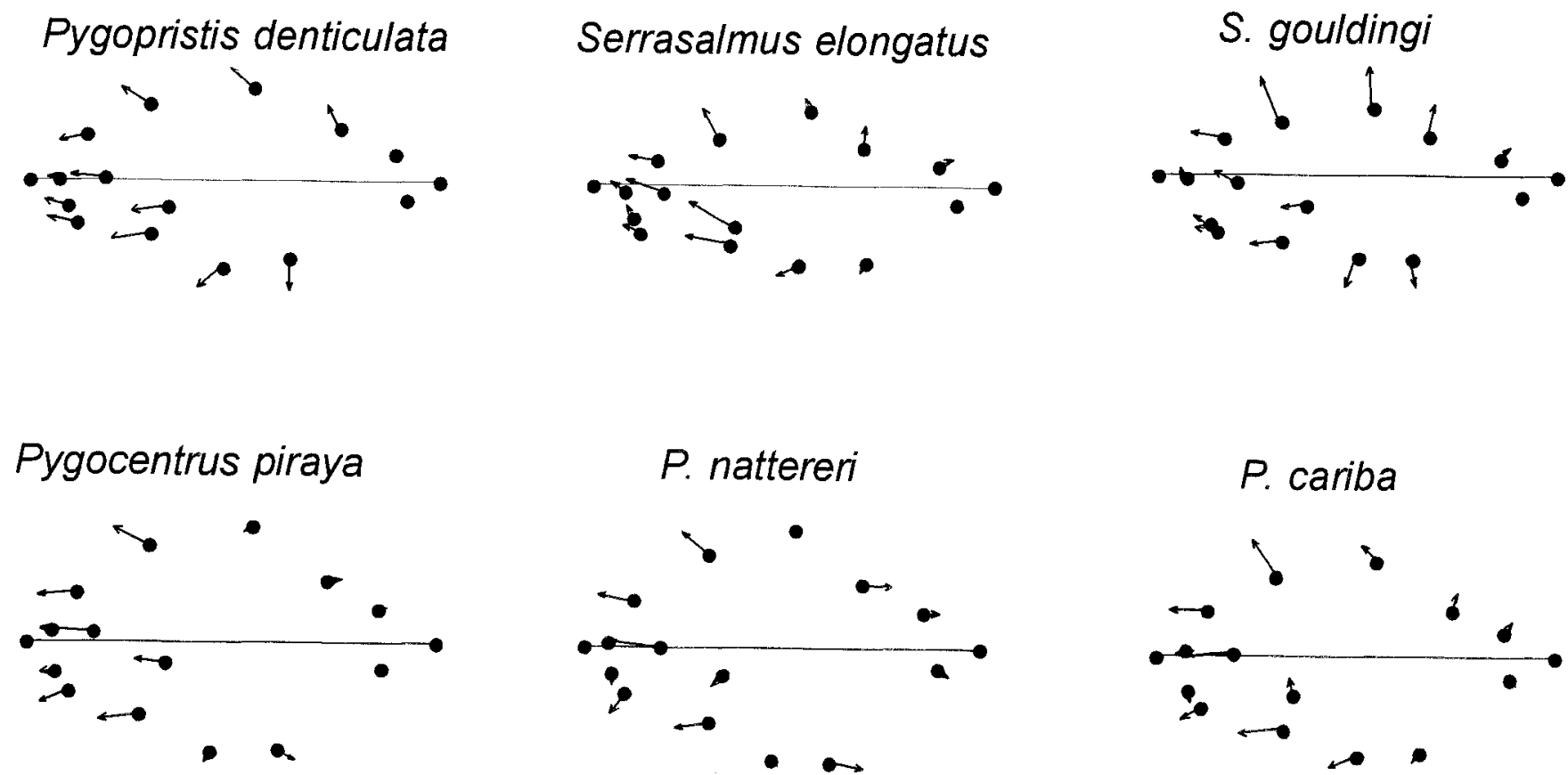

FIG. 3. Ontogenetic transformations in shape for each species. These transformations, calculated by multivariate regression of shape on size, are depicted as vectors of landmark displacements.

opment in Pygocentrus may be reduced compared to the rate in the outgroup Pygopristis; pooling the data from the three Pygocentrus species and comparing the estimated rate of the pooled samples to that of Pygopristis suggests a reduction in rate $(P=0.014)$, although this is not the case when each species of Pygocentrus is treated as a separate category in the analysis $(P=0.142)$.
The angles between Pygopristis and the other five species (Table 2) are all significantly different from zero, with the exception of the comparison between Pygopristis and S. elongatus based on the ontogenetic trajectories of shape coordinates, presumably due to the lower power of those tests compared to those based on partial warps. Also, the angle between the two species of Serrasalmus $\left(36.4^{\circ}, 30.4^{\circ}\right.$ for tra-
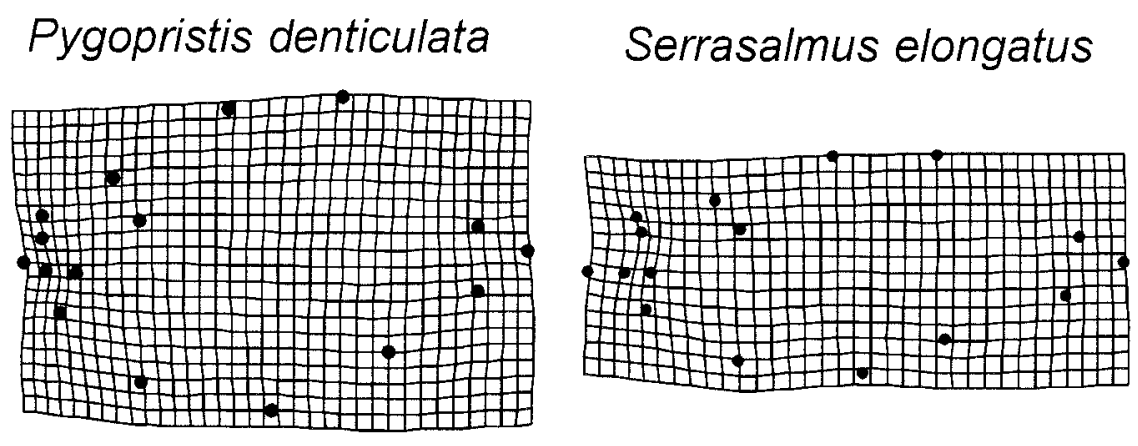

S. gouldingi

Pygocentrus piraya

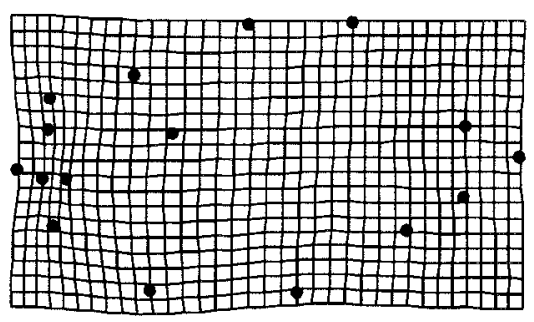

$P$. nattereri

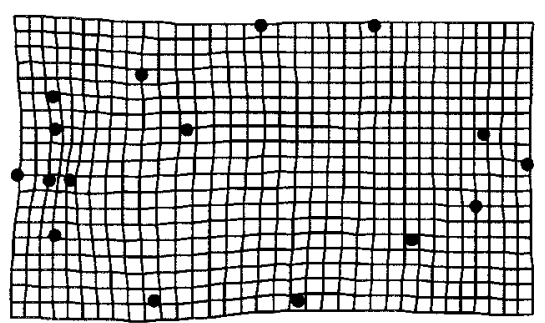

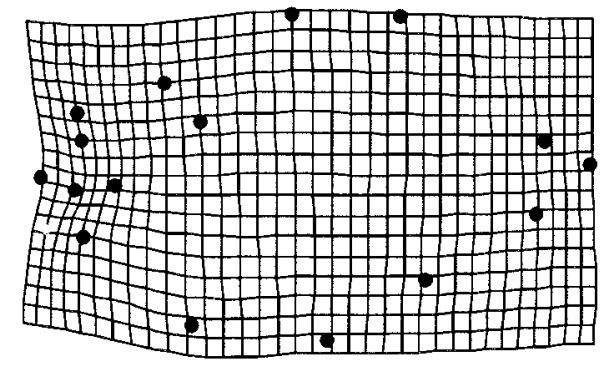

P. cariba

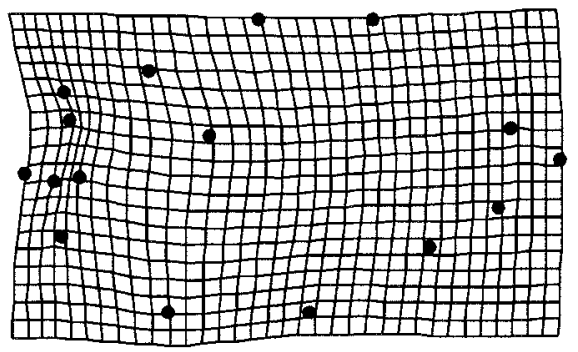

FIG. 4. Ontogenetic transformations in shape for each species. These transformations, calculated by multivariate regression of shape on size, are depicted as deformations using the thin-plate spline. 
TABLE 1. Rates of divergence away from the juvenile shape, estimated by the regression of the Procrustes distance between each specimen and the average juvenile form of its species on log-transformed centroid size.

\begin{tabular}{lccc}
\hline \hline \multicolumn{1}{c}{ Species } & Rate & SE & $R^{2}$ \\
\hline Pygopristis denticulata & 0.033 & 0.004 & 0.663 \\
Serrasalmus gouldingi & 0.059 & 0.002 & 0.950 \\
S. elongatus & 0.019 & 0.002 & 0.631 \\
Pygocentrus piraya & 0.023 & 0.002 & 0.867 \\
P. cariba & 0.024 & 0.002 & 0.856 \\
P. nattereri & 0.026 & 0.002 & 0.735 \\
\hline
\end{tabular}

jectories based on partial warps and shape coordinates, respectively) is significantly different from zero. In addition, the angles between $P$. cariba and both $P$. piraya $\left(49.4^{\circ}, 36.3^{\circ}\right.$ for trajectories based on partial warps and shape coordinates, respectively) and $P$. nattereri $\left(48.4^{\circ}, 44.2^{\circ}\right)$ are significantly different from zero $(P<0.05)$. The angle between $P$. piraya and $P$. nattereri $\left(19.1^{\circ}, 26.3^{\circ}\right.$ for trajectories based on partial warps and shape coordinates, respectively) is not significantly different from zero $(P>0.08)$.

The most striking differences between the two Serrasalmus in the ontogenetic trajectories at individual landmarks (see Figs. 3, 5) are in the vertical amplitude of the ontogenetic trajectories of landmarks at the insertion and posterior end of the dorsal fin (landmarks 4, 5, respectively), the amplitude and orientation of the vectors at the insertion of the anal, pelvic, and pectoral fins (landmark 9, 10, 11, respectively) and the horizontal amplitude of the vector at the posterior border of the bony opercle (landmark 16). There are also more subtle differences in the orientations of the vectors at the adipose fin (landmark $6 ; P=0.06$ ) and in both the amplitude and orientation at the posterior point of the eye (landmark 15). Summarizing the differences between these ontogenies over the whole configuration of landmarks (see Fig. 4), S. gouldingi deepens to a greater extent than S. elongatus, especially in the posterior region of the head (particularly suborbitally) and in the midbody, between landmarks 4 and 5 dorsally and landmarks 9 and 10 ventrally (see Fig. 4). In addition, the elongation of the postorbital region is more pronounced in S. elongatus than in S. gouldingi. Also, in $S$. gouldingi, the midbody elongates relative to the head and more caudal body, whereas in $S$. elongatus, the postcranial body elongates more smoothly, especially ventrally. In $S$. elongatus, the separation among ventral landmarks is more strictly a function of position along the anteroposterior body axis and the body elongates to an increasing extent from the head to the caudal region.

The most striking differences between $P$. nattereri and $P$. cariba are at the two landmarks of the dorsal fin (landmarks 4 and 5), the pelvic fin (landmark 10), the anterior point of the anal fin (landmark 9), and the points of the eye (landmarks 14 and 15). At two others, the trajectories at individual points differ in both amplitiude and direction, but to a less notable extent: at the adipose fin (landmark 6) and at the posterior border of the bony operculum (landmark 16). Summarizing these differences between the ontogenies over the whole configuration of landmarks (see Fig. 4), P. cariba deepens slightly more than does $P$. nattereri, but most of the difference lies in where deepening occurs. In $P$. cariba, that deepening is especially pronounced in the posterior head and anterior back, whereas in P. nattereri, the head deepens relative to the postcranial body (see Fig. 3). Additionally, in $P$. cariba there is a more pronounced deepening of the head near the level of the orbit relative to the dorsal and ventral regions than seen in $P$. nattereri. In addition, in $P$. cariba the postorbital region (and anterior postcranial body) greatly elongate, both to a greater extent than seen in $P$. nattereri and over a larger expanse of the body. Moreover, in P. nattereri the midbody elongates relative to the more caudal body (especially relative to the caudal peduncle), whereas in $P$. cariba the extent of postcranial elongation decreases posteriorly, but more smoothly than in $P$. nattereri, with a less abrupt decrease in the extent of elongation near the caudal end.

\section{DiscUSSION}

The difference in developmental rate between $S$. gouldingi and $S$. elongatus is dramatic, implicating heterochrony in the evolution of Serrasalmus. But these taxa differ in more than rate: They also differ in their ontogenetic transformations. Rather than progressing along the same ontogenetic trajectories at different rates, these species follow different ontogenetic trajectories at different rates. Interestingly, the shallow-bodied adult morphology of $S$. elongatus, which is primarily responsible for the interpretation of $S$. elongatus as neotenic (Fink 1989), is not simply a result of a decrease in overall developmental rate. It is also partly the outcome of the modified trajectory; the spatial organization of growth rates is altered so that rates of deepening increase relative to rates of growth along the anteroposterior axis. This more complex explanation for the evolution of body proportions is consistent with our conjecture that most evolutionary change occurs by a combination of heterochrony and heterotopy. That conjecture, however, is not empirically supported by the analysis of Pygocentrus. In that clade, developmental rate seems conservative. Morphological diversification of Pygocentrus, subtle as it is, apparently results solely from the evolution of spatial patterning. Contrary to our expectations, evolutionary change here results from heterotopy alone.

Our interpretations presume that the whole body can be analyzed as a single (multidimensional) feature, having a

TABle 2. Angles between the ontogenetic vector of the outgroup, Pygopristis denticulata, and the five ingroup taxa, estimated as the arc cosine of the vector correlation between ontogenetic vectors of partial warps and shape coordinates, respectively, normalized to unit length.

\begin{tabular}{lccccc}
\hline \hline & \multicolumn{4}{c}{ Species } \\
\cline { 2 - 6 } & Serrasalmus gouldingi & S. elongatus & Pygocentrus piraya & P. nattereri & P. cariba \\
\hline Angle & $33.5^{\circ}, 32.9^{\circ}$ & $36.8^{\circ}, 29.4^{\circ}$ & $55.1^{\circ}, 38.7^{\circ}$ & $65.5^{\circ}, 58.2^{\circ}$ & $58.8^{\circ}, 39.7^{\circ}$ \\
\hline
\end{tabular}




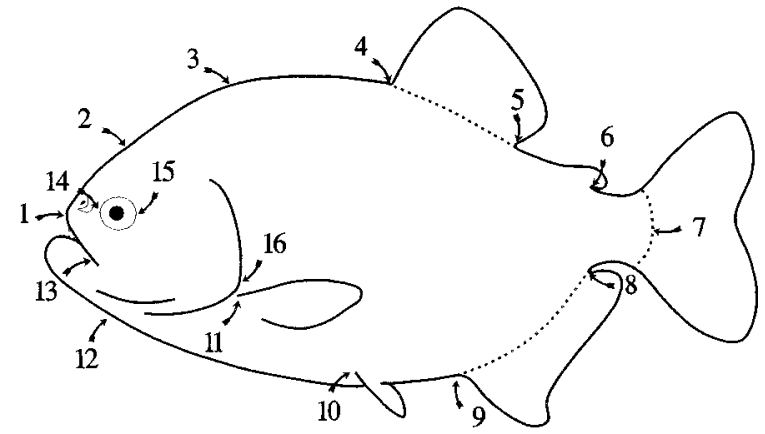

Serrasalmus gouldingi vs. S. elongatus

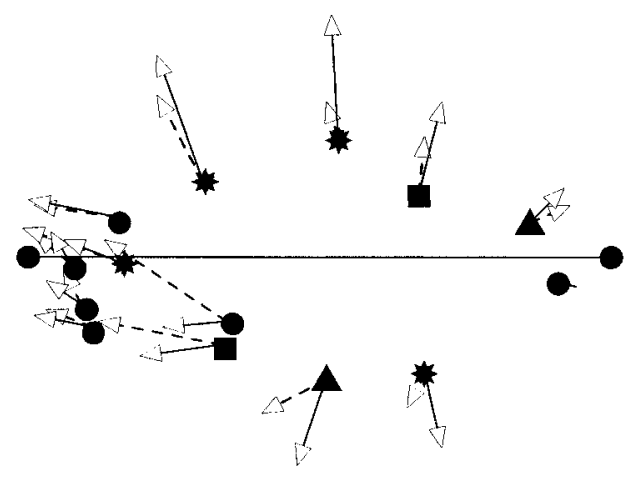

Pygocentrus cariba vs. $P$. nattereri

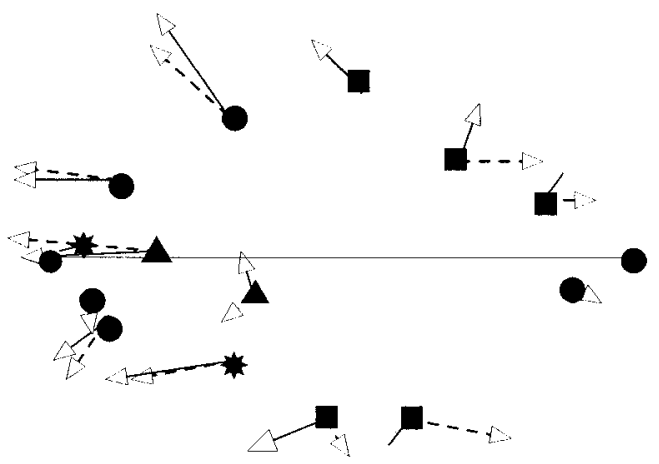

FIG. 5. Comparisons of the ontogenetic transformations in shape between Serrasalmus gouldingi and S. elongatus and between Pygocentrus cariba and P. nattereri depicted as displacements of shape coordinates. Trajectories of $S$. goulding $i$ and $P$. cariba are indicated by solid lines, those of $S$. elongatus and $P$. nattereri by dashed lines.

single ontogenetic trajectory. There are two reasons for taking this approach. First, as Gould (1977) emphasized, heterochrony is a global process that affects features of the whole organism such as age at maturity. Second, previous analyses of piranhas have focused on whole body shape, whether relating shape to diet (Nico and Taphorn 1988) or to the developmental basis of morphological evolution (Fink 1989). Considering that these hypotheses are framed in terms of overall body shape, they should be tested in those terms. However, it might seem that our interpretations ignore the possibility of dissociated heterochrony, which refers to in- dependent heterochronies of individual anatomical parts (or developmental processes) and results in a morphological mosaic of paedomorphic, peramorphic, and unmodified parts. That possibility should be considered because dissociated heterochrony is reputed to be very common (e.g., McNamara 1988) and because, like heterotopy, it can lead to divergent ontogenies. However, dissociated heterochrony does not appear to explain the divergence between species of Serrasalmus. In no well-sampled region of the body do we find anatomical parts that seem to evolve by a simple truncation or extrapolation of a shared ontogenetic trajectory. Although the ontogenetic trajectories at some landmarks of $S$. goulding $i$ are longer than those at the homologous landmarks of $S$. elongatus, there is no indication of a general acceleration of the ontogeny in any individual region. The proportions within the regions are altered, so it does not appear that any one is simply peramorphic (or paedomorphic). For the same reason, dissociated heterochrony does not seem to explain the diversification of Pygocentrus.

Dissociated heterochrony is sometimes regarded as a general rule (Fink 1982; McNamara 1988; Reilly et al. 1997), but it may be less common than often supposed. Too often, dissociation is inferred ad hoc, without evidence that individual parts actually evince heterochrony, much less that they meet the crucial assumption that the dissociated parts (or processes) are developmentally independent. If not developmentally independent, the parts do not have their own ontogenetic trajectories whose rates or timings can be shifted relative to those of other units. Heterochrony is a purely temporal dissociation; it does not, for example, lead to replacing a growth gradient with localized fields. The link between dissociated heterochrony and developmental dissociability has been stressed by several authors (e.g., Raff and Kauffman 1983; McKinney and McNamara 1991; Wagner 1996); the link is so strong that some authors explain the high frequency of heterochrony in particular groups by the modularity of their developmental system (e.g., Mosbrugger 1995). Yet, dissociation is often inferred solely because individual variables, taken separately, imply different heterochronic perturbations (e.g., McKinney 1988; McKinney and McNamara 1991). That approach is reasonable when individual variables correspond to individual parts (or processes), each of which is developmentally dissociable (at least in principle) from others. Had we adopted that approach, we could have treated each dimension of each shape coordinate or of each partial warp as a separate "part" and postulated that these undergo as many dissociated heterochronies as required to fit the data to the hypothesis. In effect, we could invoke dissociated heterochrony to account for any feature not explained by global heterochrony. In doing so, we would have ignored all other possible explanations for divergent ontogenies and transformed heterochrony from a hypothesis to a truism.

Some cases previously interpreted as dissociated heterochrony might be instances of heterotopy. Of course, we cannot generalize from our findings and conclude that heterotopy is a common phenomenon that has been generally overlooked. Yet, our findings, if not our interpretation, are consistent with numerous studies that document divergent ontogenetic trajectories (e.g., Strauss and Fuiman 1985; Klin- 
genberg and Froese 1991; Voss and Marcus 1992; Jones 1993; McLellan 1993; Bjorklund 1994; Coppinger and Schneider 1995; Monteiro et al. 1997). Other studies also suggest divergence, although the difference between the ontogenies is difficult to determine either because the ontogenetic vectors are not explicitly compared (Tissot 1988; Schweitzer and Lohmann, 1990) or because individual variables are analyzed one at a time (Wayne 1986; McNamara 1987; Geary 1988; Fiorello and German 1997). None of these studies concludes that heterotopy plays a role in the divergence of ontogeny, but none even entertains the hypothesis. The few studies that have considered heterotopy as an explanation for diversity find evidence for it (Wray and McClay 1989; Bateman 1991; Rutishauser 1997; Bradford 1998; Guralnick and Lindberg 1999). Of course, some cases of divergence might be due to dissociated heterochrony, but that hypothesis must be tested against a wider variety of alternatives than just global heterochrony.

The prevalence of heterotopy as a source of evolutionary novelty remains largely unexplored, which is surprising in light of the obvious fact that development occurs in space as well as in time. Growth rates have a location and spatial organization, not just a magnitude, and there is no good reason to suppose that spatial aspects of growth are conserved while temporal parameters evolve. Our analyses suggest that heterotopy may be more common than heterochrony, despite the perception that heterochrony is the predominant cause of morphological evolution. The prevalence of heterotopy as a source of evolutionary novelty remains largely unexplored, which is surprising in light of the obvious fact that development occurs in space as well as in time. Growth rates have a location and spatial organization, not just a magnitude, and there is no good reason to suppose that spatial aspects of growth are conserved while its temporal parameters evolve. Our analyses suggest that heterotopy may be more common than heterochrony, despite the perception that heterochrony is the predominant cause of morphological evolution. We suspect that heterochrony is seen as predominant because of concerted efforts to document it with the general neglect of alternatives. In addition, some workers have broadened the definition to encompass all conceivable possibilities, making heterochrony as common as evolution itself. Heterotopy and other alternatives to heterochrony may play major roles in the evolution of ontogeny, but that possibility cannot be judged until the search for developmental explanations looks beyond heterochrony.

\section{ACKNOWLEDGMENTS}

This research was supported in part by National Science Foundation DEB-9509195. We thank D. L. Swiderski for assistance on interpreting ontogenetic changes in shape. HDS would like to thank C. E. Mitchell (SUNYBBuffalo) for introducing him to the issues of change in shape over time.

\section{Literature Cited}

Alberch, P., and J. Alberch. 1981. Heterochronic mechanisms of morphological diversification and evolutionary change in the Neotropical salamander Bolitoglossa occidentalis (Amphibia: Plethodontidae). J. Morphol. 167:249-264.
Alberch, P., S. J. Gould, G. F. Oster, and D. B. Wake. 1979. Size and shape in ontogeny and phylogeny. Paleobiology 5:296-317.

Bateman, R. M. 1991. Paleobiological and phylogenetic implications of anatomically preserved archaeocalamites from the Dinantian of Oxroad Bay and Loch Humphrey Burn, southern Scotland. Palaeontographica B 223:1-60.

Bemis, W. 1984. Paedomorphosis and the evolution of the Dipnoi. Paleobiology 10:293-307.

Bjorklund, M. 1994. Allometric relations in 3 species of finches (Aves, Fringillidae). J. Zool. 233:657-668.

Blackstone, N. W. 1987. Size and time. Syst. Zool. 36:211-215.

Bookstein, F. L. 1986. Size and shape spaces for landmark data in two dimensions. Stat. Sci. 1:181-242

1989. Principal warps: thin-plate splines and the decomposition of deformations. IEEE T. Pattern Anal. 11:567-585.

. 1991. Morphometric tools for landmark data: geometry and biology. Cambridge Univ. Press, Cambridge, U.K.

. 1996. Combining the tools of geometric morphometrics. Pp. 131-151 in L. F. Marcus, M. Corti, A. Loy, G. J. P. Nayulor, and D. E. Slice, eds. Advances in morphometrics, Plenum Press, New York.

Bradford, J. C. 1998. A cladistic analysis of species groups in Weinmannia (Cunoniaceae) based on morphology and inflorescence architecture. Ann. Mo. Bot. Gard. 85:565-593.

Coppinger, R., and R. Schneider. 1995. Evolution of working dogs. Pp. 21-47 in J. Serpell, ed. The domestic dog: its evolution, behaviour, and interactions with people. Cambridge Univ. Press, Cambridge, U.K.

Dryden, I. L., and K. V. Mardia. 1998. Statistical shape analysis. Wiley, New York.

Edgecombe, G. D., and B. Chatterton. 1987. Heterochrony in the Silurian radiation of encrinurine trilobites. Lethaia 20:337-351.

Efron, B., and R. J. Tibshirani. 1993. An introduction to the bootstrap. Chapman and Hall, New York.

Fink, W. L. 1982. The conceptual relationship between ontogeny and phylogeny. Paleobiology 8:254-264.

1989. Ontogeny and phylogeny of shape and diet in the South American fishes called piranhas. Geobios Mem. Spec. 12: 167-172.

Fink, W. L., and M. L. Zelditch. 1995. Phylogenetic analysis of ontogenetic shape transformations: a reassessment of the piranha genus Pygocentrus (Teleostei). Syst. Biol. 44:343-360.

Fink, W. L., and M. L. Zelditch. 1996. Historical patterns of developmental integration in piranhas. Am. Zool. 36:61-69.

Fiorello, C. V., and R. Z. German. 1997. Heterochrony within species: Craniofacial growth in giant, standard and dwarf rabbits. Evolution 51:250-261.

Fuiman, L. A., L. R. Poling, and D. M. Higgs. 1998. Quantifying developmental progress for comparative studies of larval fishes. Copeia 1998:602-611.

Geary, D. 1988. Heterochrony in gastropods: A paleontological view. Pp. 183-196 in M. L. McKinney, ed. Heterochrony in evolution: a multidisciplinary approach. Plenum Press, New York.

Godfrey, L. R., and M. R. Sutherland. 1996. Paradox of peramorphic paedomorphosis: Heterochrony and human evolution. Am. J. Phys. Anthropol. 99:17-42.

Gould, S. J. 1977. Ontogeny and phylogeny. Harvard Univ. Press, Cambridge, MA.

Guralnick, R. P., and D. R. Lindberg. 1999. Integrated developmental evolutionary patterns and mechanisms: a case study using the gastropod radula. Evolution 53:447-459.

Jones, C. S. 1993. Heterochrony and heteroblastic leaf development in two subspecies of Cucurbita argyrosperma (Cucurbitaceae). Am. J. Bot. 80:778-795.

Klingenberg, C. P., and R. Froese. 1991. A multivariate comparison of allometric growth patterns. Syst. Zool. 40:410-419.

Lord, E. M., and J. P. Hill. 1987. Evidence for heterochrony in the evolution of plant form. Pp. 47-70 in R. A. Raff and E. C. Raff, eds. Development as an evolutionary process. Alan R. Liss, Inc, New York.

Mathworks. 1997. MATLAB. Vers. 5.1.0.The Mathworks, Natick, MA. 
McKinney, M. L. 1988. Classifying heterochrony: Allometry, size, and time. Pp. 17-34 in M. L. McKinny, ed. Heterochrony in evolution: a multidisciplinary approach. Plenum, New York.

McKinney, M. L., and K. J. McNamara. 1991. Heterochrony: the evolution of ontogeny. Plenum Press, New York.

McLellan, T. 1993. The roles of heterochrony and heteroblasty in the diversification of leaf shapes in Begonia dregei (Begoniaceae). Am. J. Bot. 80:796-804.

McNamara, K. J. 1978. Paedomorphosis in Scottish olenelid trilobites (Early Cambrian). Palaeontology 21:635-655.

. 1987. Plate translocation in spatangoid echinoids: its morphological, functional and phylogenetic significance. Paleobiology 13:312-325.

. 1988. The abundance of heterochrony in the fossil record. Pp. 287-325 in M. L. McKinney, ed. Heterochrony in evolution: a multidisciplinary approach. Plenum Press, New York.

Monteiro, L. R., M. J. Cavalcanti, and H. J. S. Sommer III. 1997. Comparative ontogenetic shape change in the skull of Caiman species (Crocodylia, Alligatoridae). J. Morphol. 231:53-62.

Mosbrugger, V. 1995. Heterochrony and the evolution of land plants. Pp. 93-105 in K. J. McNamara, ed. Evolutionary change and heterochrony. Wiley, New York.

Nico, L. G., and D. C. Taphorn. 1988. Food habits of piranhas in the low llanos of Venezuela. Biotropica 20:311-321.

Raff, R. A., and T. C. Kaufman. 1983. Embryos, genes, and evolution. Indiana Univ. Press, Bloomington.

Ramskold, L. 1988. Heterochrony in Silurian phacopid trilobites as suggested by the ontogeny of Acernaspis. Lethaia 21:307-318.

Reilly, S. M., E. O. Wiley, and D. J. Meinhardt. 1997. An integrated approach to heterochrony: the distinction between interspecific and intraspecific phenomena. Biol. J. Linn. Soc. 60:119-143.

Rohlf, F. J. 1998a. On applications of geometric morphometrics to studies of ontogeny and phylogeny. Syst. Biol. 47:147-158.

1998b. tpsRegr vers. 1.17. State Univ. of New York, Buffalo, NY.
Rutishauser, R. 1997. Structural and developmental diversity in Podostemaceae (river-weeds). Aquat. Bot. 57:29-70.

Schweitzer, P. N., and G. P. Lohmann. 1990. Life-history and the evolution of ontogeny in the ostracode genus Cypridies. Paleobiology 16:107-125.

SPSS. 1990. Systat 5.05 for Windows. SPSS, Inc. Evanston, IL.

Strauss, R. E. 1987. On allometry and relative growth in evolutionary studies. Syst. Zool. 36:72-75.

Strauss, R. E., and L. A. Fuiman. 1985. Quantitative comparisons of body form and allometry in larval and adult Pacific sculpins (Teleostei: Cottidae). Can. J. Zool. 63:1582-1589.

Swiderski, D. L. 1993. Morphological evolution of the scapula in tree squirrels, chipmunks, and ground squirrels (Sciuridae): an analysis using thin-plate splines. Evolution 47:1854-1873.

Tissot, B. N. 1988. Geographic variation and heterochrony in two species of cowries (genus Cypraea). Evolution 42:103-117.

Voss, R. S., and L. F. Marcus. 1992. Morphological evolution in muroid rodents. 2. Craniometric factor divergence in 7 neotropical genera, with experimental results from Zygodontomys. Evolution 46:1918-1934.

Wagner, G. P. 1996. Homologues, natural kinds and the evolution of modularity. Am. Zool. 36:36-43.

Wayne, R. 1986. Cranial morphology of domestic and wild canids: the influence of development on morphological change. Evolution 40:243-261.

Winterbottom, R. 1990. The Trimmatom nanus species complex (Actinopterygii, Gobiidae). Syst. Zool. 39:253-265.

Wray, G. A., and D. R. McClay. 1989. Molecular heterochronies and heterotopies in early echinoid development. Evolution 43: 803-813.

Zelditch, M. L., and W. L. Fink. 1995. Allometry and developmental integration of body growth in a piranha, Pygocentrus nattereri (Teleostei, Ostariophysi). J. Morphol. 223:341-355.

1996. Heterochrony and heterotopy: stability and innovation in the evolution of form. Paleobiology 22:241-254.

Corresponding Editor: J. Losos 